\title{
FIELD MEASUREMENT RESULTS OF THE KEK B-FACTORY QUADRUPOLE AND SEXTUPOLE MAGNETS
}

\author{
K.Egawa, M.Masuzawa ${ }^{\#}$, KEK, Tsukuba, Japan
}

\section{Abstract}

The magnetic properties of the quadrupole and sextupole magnets for the KEK B-factory rings (LER and HER)[1] are measured by two sets of harmonic coil systems, one for the LER magnets and the other for the HER magnets, at KEK over an 18 month period[2]. The main field strength, offsets between the mechanical and magnetic centers, higher order multipole components and effective lengths of the magnets are measured.

\section{INTRODUCTION}

The design parameters of the quadrupole and sextupole magnets are given in Table 1[1]. QA(HER) and QB(HER) are recycled TRISTAN magnets[3]. QA(LER), Qs(HER), Qx(HER), QA(HER) and QB(HER) are the quadrupole magnets in the arc sections. Qrf(LER) and Qrf(HER) are the quadrupole magnets for the straight sections where the RF stations are located[1]. The skew quadrupole magnets are Qsk(LER), Qsk1(HER), and Qsk2(HER). The remaining magnets in the table are the sextupole magnets. The field gradient in the table is calculated at the maximum current of the power supplies used. The maximum current values are $200 \mathrm{~A}$ for the skew quadrupoles, 500A for the other quadrupoles and 425A for the sextupoles. All of the magnets are fabricated from 0.5 mm-thick lamination plates. The specified tolerance for the core assembly is $0.35 \mathrm{~mm}[3]$.

Table 1: Magnet parameters

\begin{tabular}{|l|l|l|l|l|}
\hline Magnet & $\begin{array}{l}\mathrm{r} \\
(\mathrm{mm})\end{array}$ & $\begin{array}{l}\mathrm{L} \\
(\mathrm{mm})\end{array}$ & $\begin{array}{l}\mathrm{B}^{\prime}(\mathrm{T} / \mathrm{m}) \text { or } \\
\mathrm{B}^{\prime}\left(\mathrm{T} / \mathrm{m}^{2}\right)\end{array}$ & $\begin{array}{l}\text { \# of } \\
\mathrm{mag}\end{array}$ \\
\hline QA(LER) & 55 & 400 & 10.2 & 414 \\
\hline Qrf(LER) & 83 & 500 & 6.3 & 36 \\
\hline Qsk(LER) & 105 & 200 & 0.7 & 8 \\
\hline Sx(LER) & 56 & 300 & 340 & 104 \\
\hline SxC(LER) & 85 & 300 & 77.5 & 4 \\
\hline Qs(HER) & 50 & 500 & 12.7 & 80 \\
\hline Qx(HER) & 50 & 760 & 12.7 & 4 \\
\hline Qrf(HER) & 83 & 1000 & 6.3 & 43 \\
\hline Qsk1(HER) & 105 & 500 & 0.7 & 7 \\
\hline Qsk2(HER) & 80 & 300 & 1.3 & 5 \\
\hline QA(HER) & 50 & 762 & 8.5 & 199 \\
\hline QB(HER) & 50 & 960 & 8.5 & 110 \\
\hline SxF(HER) & 56 & 300 & 340 & 56 \\
\hline SxD(HER) & 56 & 1000 & 350 & 48 \\
\hline
\end{tabular}

Note: $\mathrm{r}$ and $\mathrm{L}$ denote the bore radius and the lamination length, respectively.

\footnotetext{
\# Email: mika@ post.kek.jp
}

\section{HARMONIC COIL MEASUREMENTS}

\subsection{Quadrupole Magnets}

The integrated quadrupole field along the beam line (Zaxis) is measured by a harmonic coil, $1.5 \mathrm{~m}$ in length for the LER system and $1.8 \mathrm{~m}$ for the HER system. The dipole components measured by $0.2 \mathrm{~m}$ short coils are used to calculate the transverse offset between the mechanical and magnetic centers. Figs. 1(a)-(c) show the field strength distributions at various currents for QA(LER). Fig. 1(d) gives the distribution of the ratio of the central field measured by the central short coil to the integrated field measured by the long coil. This is proportional to the effective length of the magnet. Figs. 1(a)-(e) present the corresponding distributions for different currents. The standard deviation has a slight dependence on the field strength. This is due to the iron permeability characteristics at different field strengths. Fig. 2 shows the normalized higher order multipole components for QA(LER). A pole-end shim correction is made in order to reduce the n6 component. The offsets between the mechanical and magnetic centers are shown in Fig. 3 for $\mathrm{QA}(\mathrm{LER})$. $\mathrm{X}, \mathrm{y}$ and $\mathrm{z}$ correspond to the horizontal, vertical and beam directions, respectively. The measurement results are summarized in Table 2 for the quadrupole magnets.

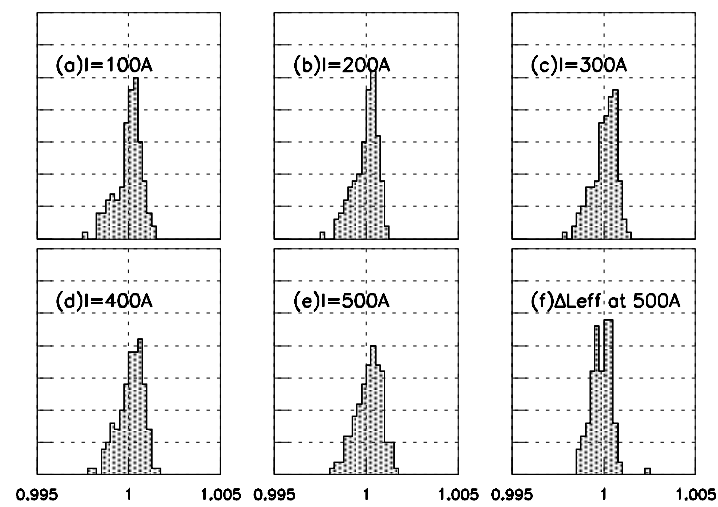

Figure 1: QA(LER) quadrupole field strength distribution at

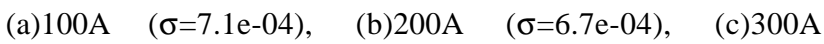
$(\sigma=6.7 \mathrm{e}-04)$, (d)400A $(\sigma=7.2 \mathrm{e}-04)$ and $(\mathrm{e}) 500 \mathrm{~A}(\sigma=7.2 \mathrm{e}-04)$. The integrated quadrupole field is normalized by the average value. Fig. 1(f) represents the distribution of the effective length at $\mathrm{I}=500 \mathrm{~A}(\sigma=5.7 \mathrm{e}-04)$. 


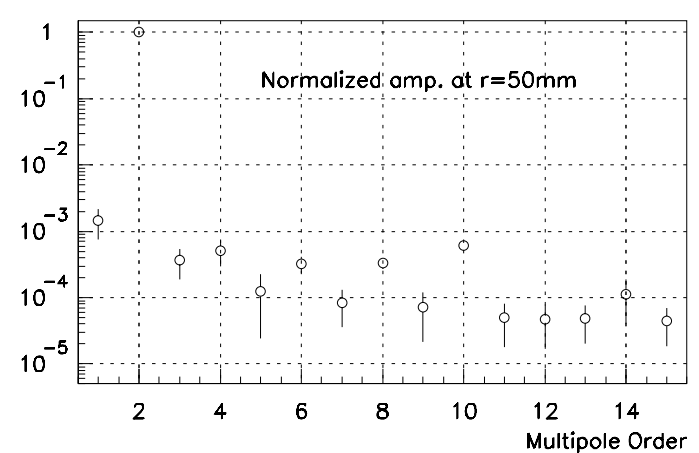

Figure 2: Normalized amplitude is plotted against harmonic numbers for QA(LER). Error bars correspond to one standard deviation.

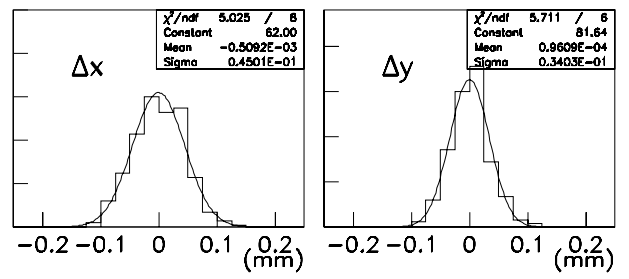

Figure 3: The horizontal and vertical offsets between the mechanical and magnetic centers for QA(LER).

Table 2: Measurement Results for the Quadrupoles

\begin{tabular}{|l|l|l|l|l|}
\hline Magnet & $\Delta \mathrm{B}^{\prime} \mathrm{L}$ & $\Delta \mathrm{x}$ & $\Delta \mathrm{y}$ & $\Delta \mathrm{L}_{\text {eff }}$ \\
\hline QA(LER) & $7.2 \mathrm{e}-04$ & 50 & 30 & $4.5 \mathrm{e}-04$ \\
\hline Qrf(LER) & $6.8 \mathrm{e}-04$ & 30 & 30 & $3.5 \mathrm{e}-04$ \\
\hline Qsk(LER) & $7.1 \mathrm{e}-04$ & 50 & 70 & $7.0 \mathrm{e}-04$ \\
\hline Qs(HER) & $4.9 \mathrm{e}-04$ & 60 & 50 & $6.0 \mathrm{e}-04$ \\
\hline Qx(HER) & $7.5 \mathrm{e}-04$ & 60 & 10 & $2.0 \mathrm{e}-04$ \\
\hline Qrf(HER) & $5.5 \mathrm{e}-04$ & 50 & 40 & $3.8 \mathrm{e}-04$ \\
\hline Qsk1(HER) & $3.1 \mathrm{e}-04$ & 30 & 30 & $4.2 \mathrm{e}-04$ \\
\hline Qsk2(HER) & $2.1 \mathrm{e}-04$ & 40 & 10 & $2.7 \mathrm{e}-04$ \\
\hline QA(HER) & $6.0 \mathrm{e}-04$ & 40 & 70 & $4.4 \mathrm{e}-04$ \\
\hline QB(HER) & $5.0 \mathrm{e}-04$ & 50 & 60 & $4.2 \mathrm{e}-04$ \\
\hline
\end{tabular}

$\Delta \mathrm{B}^{\prime} \mathrm{L}$ is the RMS of the normalized quadrupole field distribution at the field strength given in Table $1 . \Delta \mathrm{x}$ and $\Delta y$ are the horizontal and vertical offsets in $\mu \mathrm{m}$ between mechanical and magnetic centers. $\Delta \mathrm{L}_{\text {eff }}$ is the RMS of the effective length.

\subsection{Sextupole Magnets}

The sextupole magnets are measured with the same system as the quadrupole magnets. The normalized integrated sextupole field is plotted against the magnet production number in Fig. 4. The lamination punching dye was modified after the first $\sim 40$ magnets. There is a noticeable effect as is shown in the plot. According to the field strength, all of the sextupole magnets are paired so as to keep the difference between the pair below 5.e-04. The sextupole field strength errors are shown in Figs. 5(a)-(d) for SxD. Fig. 5(d) presents the remnant field distribution, which reflects the variation of the coercive force of the iron. The higher order multipole components normalized to the sextupole strength at $\mathrm{I}=425 \mathrm{~A}$ is plotted in Fig. $6(\mathrm{a})$ for $\operatorname{SxD}(\mathrm{HER})$ and (b) for $\mathrm{SxC}(\mathrm{LER})$. The other measurement results for the sextupole magnets are given in Table 3.

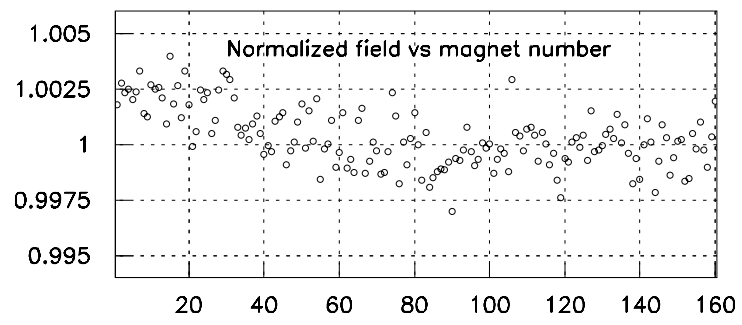

Figure 4: Normalized (by the average) integrated sextupole field plotted against the production number of the magnets.
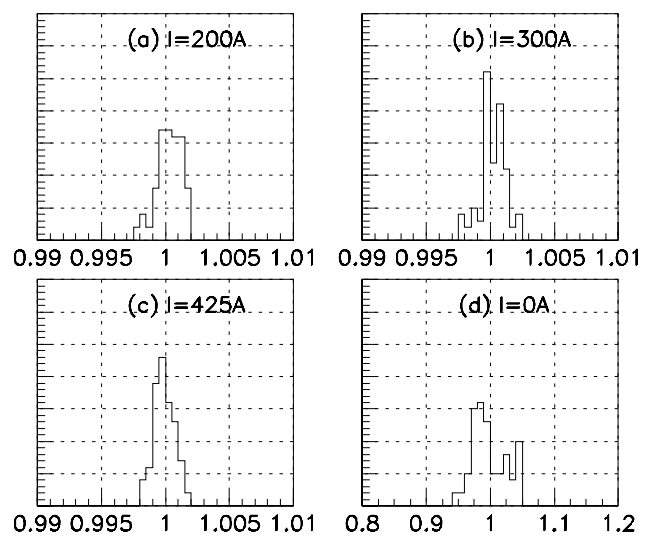

Figure 5: The sextupole field strength distribution at (a) $I=200 \mathrm{~A}$, (b) $\mathrm{I}=300 \mathrm{~A}$, (c) $\mathrm{I}=425 \mathrm{~A}$ and (d) $\mathrm{I}=0 \mathrm{~A}$ for $\mathrm{SxD}$. The field is normalized by the average for each current. The RMSs are (a)9.7e-04, (b)1.0e-03, (c)8.0e-04 and (d)2.7e-02.
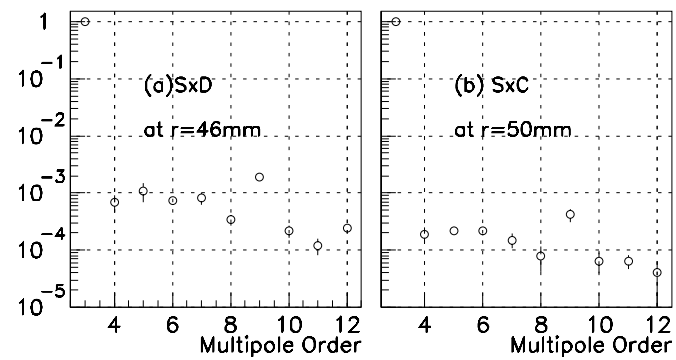

Figure 6: Higher order multipole components at I=425A for (a) $\mathrm{SxD}$ (HER) and (b)SxC(LER). A larger bore radius results in the better field quality of $\mathrm{SxC}(\mathrm{LER})$.

Table 3: Measurement Results for the Sextupoles

\begin{tabular}{|l|l|l|l|}
\hline Magnet & $\Delta \mathrm{B}^{\prime \prime} \mathrm{L}$ & $\Delta \mathrm{x}(\mu \mathrm{m})$ & $\Delta \mathrm{y}(\mu \mathrm{m})$ \\
\hline Sx(LER),SxF(HER) & $1.3 \mathrm{e}-03$ & 30 & 40 \\
\hline SxC(LER) & $3.3 \mathrm{e}-04$ & 20 & 20 \\
\hline SxD(HER) & $8.1 \mathrm{e}-04$ & 40 & 40 \\
\hline
\end{tabular}




\section{MAPPING}

A mapping is performed for one magnet in each group. The system consists of a twin-flip coil[4]. The coil moves along the Z-direction and the field is measured every 10mm. Fig. 7 and Fig. 8 are the field distribution along the Z-direction for Qrf(LER) and QB(HER). Table 4 summarizes the effective lengths of the quadrupole and sextupole magnets at the maximum field strengths. The $\mathrm{L}_{\text {eff }}$ dependence on the current (field strength) is plotted for various types of LER and HER quadrupole magnets in Figs. 9(a) and (b).

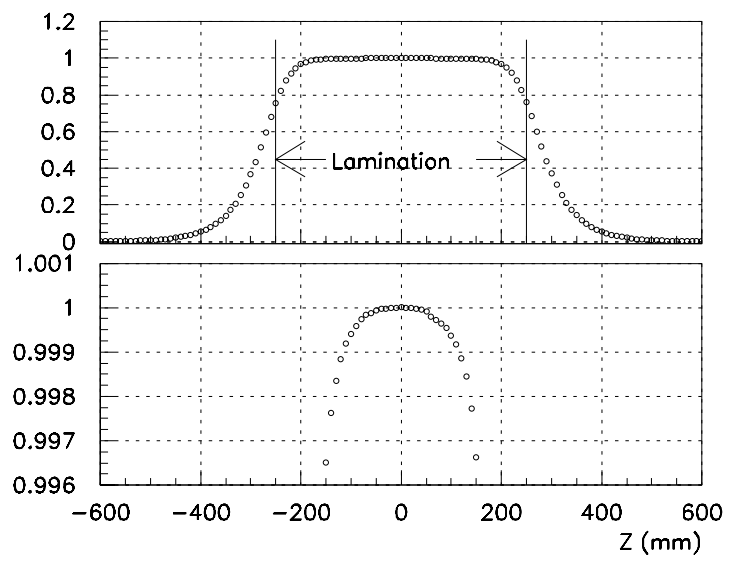

Figure 7: Qrf(LER) mapping results. The top plot shows the entire mapping region while the bottom one shows the central region only (fine scale). The magnet center corresponds to $\mathrm{Z}=0$.

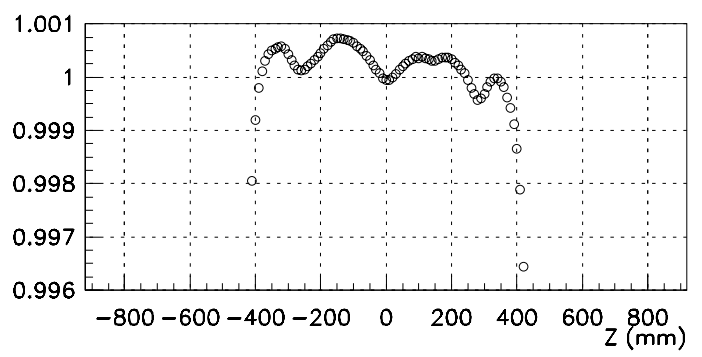

Figure 8: $\mathrm{QB}(\mathrm{HER})$ fine scale mapping results. The distribution indicates an uneven lamination stacking or/and a distortion from welding.

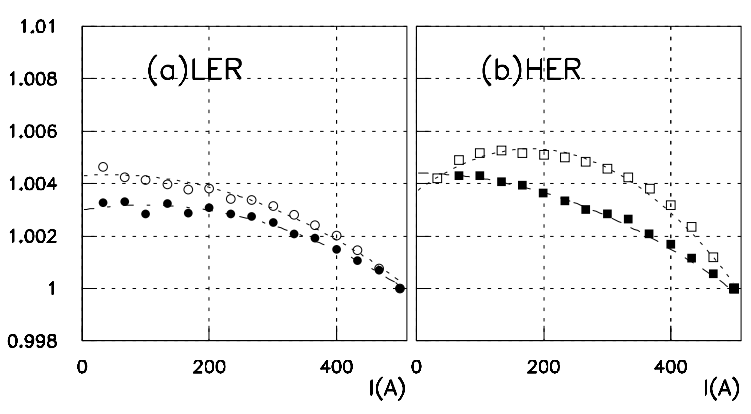

Figure 9: Normalized $\mathrm{L}_{\text {eff }}$ as a function of $\mathrm{I}_{\mathrm{L}} \mathrm{L}_{\text {eff }}$ is normalized by the values in Table 4 . Solid circle, open circle, solid square and open square correspond to QA(LER), Qrf(LER), QS(HER) and Qrf(HER), respectively.
Table 4: Effective Lengths calculated from Mapping

\begin{tabular}{|l|l|l|l|}
\hline Magnet & $\mathrm{L}_{\text {eff }}(\mathrm{mm})$ & Magnet & $\mathrm{L}_{\text {eff }}(\mathrm{mm})$ \\
\hline QA(LER) & 458.987 & Qrf(HER) & 1079.31 \\
\hline Qrf(LER) & 582.129 & Qsk1(HER) & 593.12 \\
\hline Qsk(LER) & 297.802 & Qsk2(HER) & 379.86 \\
\hline Sx(LER) & 355.795 & QA(HER $)$ & 825.42 \\
\hline SxC(LER) & 552.767 & QB(HER) & 1015.51 \\
\hline Qs (HER) & 554.102 & SxF(HER $)$ & 355.80 \\
\hline Qx(HER) & 812.589 & SxD(HER $)$ & 1019.13 \\
\hline
\end{tabular}

\section{CALIBRATION}

The harmonic coil system is calibrated with a dipole magnet of known integrated dipole field strength, BL. BL is translated into the radius of the harmonic coil and thus the calibration constant is obtained. The calibration constants obtained from this method, however, are still preliminary. Calibration with other methods is needed in order to determine the quadrupole and sextupole field strengths to within 1.e-03 accuracy.

\section{SUMMARY}

Series measurements of the KEK B-factory LER and HER quadrupole and sextupole magnets are completed. The quality of the newly fabricated magnets and the recycled TRISTAN magnets have been checked. The RMSs of the offsets between the mechanical and magnetic centers are smaller than the beam optics requirements of $0.1 \mathrm{~mm}$ in both $\mathrm{x}$ and $\mathrm{y}$ directions. The RMS of the field strength distributions, the magnitude of the higher order multipole components and the strength of the main field also satisfy the optics requirements.

\section{ACKNOWLEDGMENTS}

The authors would like to thank the measurement crew for their hard work, the KEK magnet group for many useful discussions.

\section{REFERENCES}

[1] KEKB B-Factory Design Report 95-7.

[2] K. Egawa and M. Masuzawa, "Preliminary Results of the KEKB Quadrupole Magnet Measurements", EPAC'98, Stockholm (1998).

[3] "TRISTAN electron-positron colliding beam project", KEK Report 6-24 March 1987.

[4] K.Egawa and M.Masuzawa, "Field Measurements of the KEK B-Factory Dipole and Wiggler Magnets", Contribution to PAC99. 